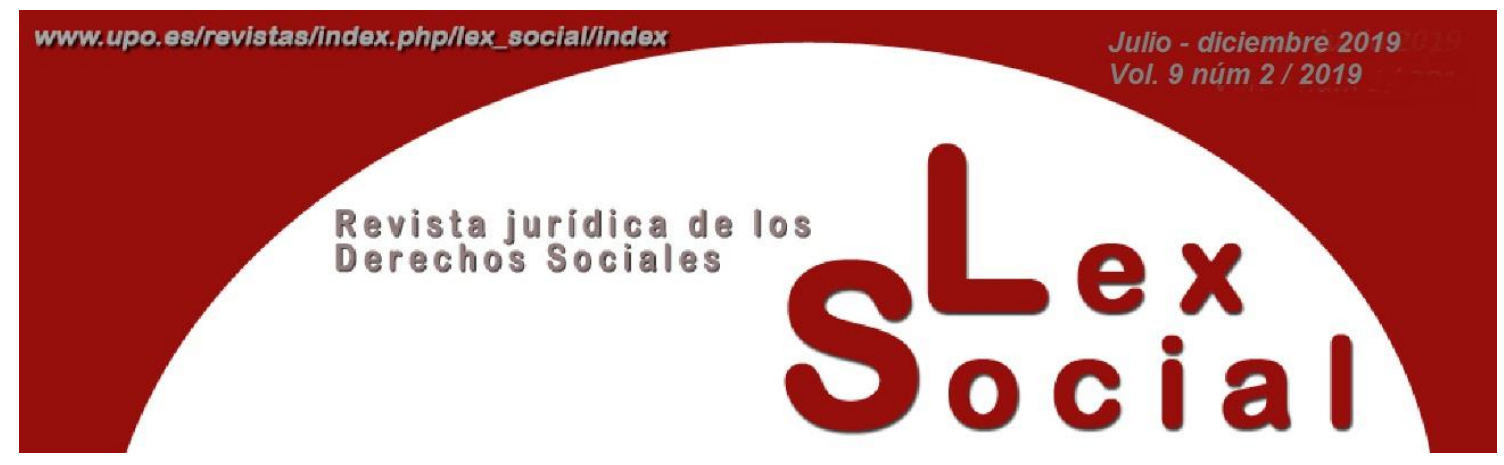

\title{
La contratación irregular de los empleados del servicio del hogar familiar
}

\section{The irregular hiring of the employees of the family home service}

\author{
MARIA JOSÉ RODRIGUEZ CRESPO \\ Departamento de Derecho del Trabajo \\ Universidad de Córdoba
}

Artículo recibido el 8 de mayo de 2019

Artículo aceptado el 27 de mayo de 2019

\begin{abstract}
RESUMEN
La precariedad laboral que caracteriza el trabajo doméstico tiene en la contratación irregular de los empleados/as de hogar uno de sus pilares. El ingreso irregular en el mercado laboral se está viendo favorecido por la aparición de nuevos agentes implicados en la contratación de estos trabajadores que, en ocasiones, actúan al margen de la legalidad. El presente estudio se centra, en primer lugar, en el análisis de los supuestos problemáticos que giran actualmente en torno a la contratación irregular, ya sea por la externalización de actividades de trabajo doméstico a través de empresas de servicios o por la realización de prácticas abusivas que degeneran en precariedad laboral. En segundo lugar, se delimitan las consecuencias que para los implicados (empleados y empleadores) tienen las actuaciones realizadas. Y, en tercer lugar, se proponen mejoras legislativas que permitan eliminar los abusos que supone la contratación irregular de estos trabajadores.
\end{abstract}

Palabras Clave: contratación irregular, intermediación, cesión ilegal de trabajadores, empleados de hogar. 


\begin{abstract}
The precarious work that characterizes domestic work has in the irregular hiring of domestic workers one of its pillars. Irregular entry into the labor market is being favored by the appearance of new agents involved in the hiring of these workers, who sometimes act outside the law. The present study focuses, first, on the analysis of the problematic assumptions currently revolving around irregular hiring, either by outsourcing domestic work activities through service companies or by carrying out abusive practices that degenerate into job insecurity. Secondly, the consequences that for those involved (employees and employers) have the actions taken are defined. And, thirdly, legislative improvements are proposed to eliminate the abuses implied by the irregular hiring of these workers.
\end{abstract}

KEYWORD: irregular hiring, intermediation, illegal assignment of workers, household employees

\title{
SUMARIO
}

1. La contratación de los empleados al servicio del hogar familiar y su problemática.

2. La contratación directa y regular de los empleados al servicio del hogar familiar.

3. La contratación mediante la intermediación de los servicios públicos de empleo o de las agencias de colocación.

4. La externalización de actividades mediante empresas de servicios domésticos y la contratación irregular de los empleados al servicio del hogar familiar.

4.1. Estado de la cuestión.

4.2. Análisis de los supuestos problemáticos en torno a la contratación irregular por parte de las empresas de servicios.

5. Conclusiones y propuestas para mejorar la contratación irregular de los trabajadores al servicio del hogar familiar.

BIBLIOGRAFÍA

\section{LA CONTRATACIÓN DE LOS EMPLEADOS AL SERVICIO DEL HOGAR FAMILIAR Y SU PROBLEMÁTICA.}

Trabajo doméstico, precarización y explotación son palabras que actualmente se nos antojan sinónimas a la luz de los informes presentados desde instancias internacionales en los que se aboga por seguir trabajando para asegurar que los 
trabajadores domésticos estén protegidos por la legislación laboral y existan recursos efectivos y mecanismos de denuncia por abusos por los empleadores ${ }^{1}$.

En nuestro país, la reforma operada por aprobación del Real Decreto 1602/2011, de 14 de noviembre, por el que se regula actualmente la relación laboral de carácter especial del servicio del hogar familiar, tuvo como objetivo dignificar las condiciones laborales del sector doméstico acercándolo a la normativa existente para los demás sectores. Si bien en un primer momento, se produjo un aumento de las afiliaciones y la regulación de los trabajadores del sector, a partir de 2015 la regulación se ha tornado decreciente $^{2}$, con un aumento de la informalidad, en la que la aparición de agencias de colocación no oficiales son parte del problema. Junto a las clásicas causas de precariedad que caracterizan al trabajo doméstico (consideración social del trabajo doméstico como tradicionalmente asignado al sexo femenino, lugar de la prestación del servicio, etc.), aparecen otras ligadas a la intermediación laboral por la actuación de agentes intermediarios que actúan en ocasiones fuera de la ley o constituidos directamente como mafias, lo que genera situaciones de explotación laboral e incluso de explotación humana ${ }^{3}$.

Tradicionalmente, el ingreso en el mercado laboral de los trabajadores al servicio del hogar familiar no ha presentado especiales dificultades, dado que la formalización se realizaba de forma directa, entre el empleador, titular del hogar familiar y la persona que, dependientemente y por cuenta de aquél, había de realizar los servicios retribuidos en el ámbito del hogar familiar. Así lo disponía el artículo 2 del ya derogado Real Decreto 1424/1985, de 1 de agosto ${ }^{4}$. Y así también lo establece el art. 1.2 del Real Decreto 1620/2011, de 14 de noviembre, por el que se regula actualmente la relación laboral de carácter especial del servicio del hogar familiar, pero la aparición en el mercado laboral de nuevos agentes implicados en la contratación de los empleados de hogar, como son las agencias de colocación, las empresas de trabajo temporal y las empresas de servicios domésticos, han determinado un aumento de la complejidad y conflictividad en este sentido. Se han detectado abusos por parte de las agencias de colocación en la contratación de los empleados de hogar, debido fundamentalmente a su actuación como agencias de servicio y no como meros intermediadores del mercado laboral. A ello se une la posibilidad abierta por la disposición adicional $17^{\mathrm{a}}$ de la Ley 27/2011 de permitir a las empresas de servicios la contratación de empleados de hogar, con la consiguiente inseguridad jurídica que ello determina. En la práctica, es esta

\footnotetext{
${ }^{1}$ Naciones Unidas. Informe de la Relatora Especial sobre las formas contemporáneas de la esclavitud, incluidas sus causas y consecuencias, Sra. Gulnara Shahinian. 2010; OIT. Seguimiento de la resolución sobre medidas encaminadas a hacer realidad el trabajo decente para las trabajadoras y los trabajadores domésticos en todo el mundo: informe sobre los progresos realizados. 2013.

${ }^{2}$ OIT. Informe "Impacto de las reformas legislativas en el sector del empleo del hogar en España". 2016.

${ }^{3}$ Lousada Arochena, J. F. Normativa internacional contra la explotación humana y laboral en el trabajo doméstico: la ONU y la OIT. Lan Harremanak. Nº 39. 2018, pág. 157.

4 “Dos.- Se considera relación laboral especial del servicio del hogar familiar la que conciertan el titular del mismo, como empleador, y la persona que, dependientemente y por cuenta de aquel, presta servicios retribuidos en el ámbito del hogar familiar. Real Decreto 1424/1985, de 1 de agosto, por el que se regula la relación laboral de carácter especial del Servicio del Hogar Familiar". 
última opción la que está generando mayor conflictividad debido al auge de empresas o agencias de servicios e incluso plataformas de economía colaborativa, a través de las cuales, bajo la mera apariencia de la contratación de empleados del servicio doméstico, se esconden prácticas abusivas que conducen a la precarización del empleo y de las condiciones laborales de las personas empleadas.

La solución de las controversias que giran en torno a la contratación irregular de trabajadores al servicio del hogar familiar parte del análisis primigenio de la legislación que regula el acceso al mercado laboral de estos trabajadores, puntualizando la labor que juegan los agentes intermediadores en este sentido. El análisis posterior de los supuestos problemáticos que giran actualmente en torno a la contratación irregular ya sea por la externalización de actividades de trabajo doméstico a través de empresas de servicios o por la realización de prácticas abusivas que degeneran en precariedad laboral, nos permitirá extraer consecuencias en torno a los derechos laborales (su amplitud, sus garantías, etc.), para, ulteriormente, proponer en la medida de lo posible mejoras legislativas que permitan eliminar los abusos que supone la contratación irregular de estos trabajadores.

\section{LA CONTRATACIÓN DIRECTA Y REGULAR DE LOS EMPLEADOS AL SERVICIO DEL HOGAR FAMILIAR.}

En la relación especial de los empleados al servicio del hogar familiar el elemento subjetivo es determinante a la hora de concretar cuáles son los sujetos que intervienen cuáles son las relaciones que pueden surgir entre ellos. De forma clara, el artículo 4.1 del Real Decreto 1620/2011, de 14 de noviembre, dispone que "los empleadores podrán contratar a los trabajadores directamente o por intermediación de los servicios públicos de empleo o de agencias de colocación debidamente autorizadas". La delimitación de quiénes pueden ser empleadores determina, en buena medida, la eliminación de supuestos fraudulentos al inicio de la relación laboral. Es por ello por lo que debe ser objeto de análisis prioritario a las formas de contratación regulares e irregulares, toda vez que el contrato especial del servicio de hogar es una excepción al régimen común y general del contrato de trabajo, tal y como establece el art. 2.1 b) del Estatuto de los Trabajadores. La característica de esta relación especial se acentúa y define por el legislador de manera que se excluyen del régimen general, por lo que pueden existir particularidades en torno a la figura del empleador que puede no coincidir con el establecido de manera general en el art. 1.2 del Estatuto de los Trabajadores. De ahí que sea necesario buscar esa naturaleza diferenciadora del servicio del hogar para poder definir, evaluar y catalogar la vinculación que existe entre empleador y empleado, pues sólo cuando concurran los requisitos y presupuestos previstos en la legislación nos encontraremos ante una contratación regular. 
En esta tesitura de diferenciar el contrato especial del común, el referido al elemento subjetivo es el que muestra la peculiaridad específica de la contratación del servicio de hogar familiar. Así, se considerará empleador "al titular del hogar familiar, ya lo sea efectivamente o como simple titular del domicilio o lugar de residencia en el que se presten los servicios domésticos", según dispone el art. 1.3 del Real Decreto 1620/2011. También se considerará como tal, en el caso de que dos o más personas convivan sin constituir una familia ni una persona jurídica, quien ostente la titularidad de la vivienda o asuma la representación de tales personas (art. 1.3 del Real Decreto 1620/2011). Dicho elemento subjetivo se conforma a su vez de forma dual: por el espacio o lugar en el que se lleva a cabo el servicio y por el tipo de relación o vinculación personal que se mantiene en el ámbito donde se lleva a cabo la prestación de servicios ${ }^{5}$. El elemento del "hogar familiar" es el eje sobre el que gravitan todas las coyunturas específicas de esta contratación: personas y lugares; tal y como se define en el art. 1.2 del Real Decreto 1620/2011 cuando alude al ámbito del hogar familiar, reafirmándose en el objeto de la relación laboral al ser éste los servicios o actividades prestados para el hogar familiar (art. 1.4 del Real Decreto 1620/2011). Este elemento característico ya fue inicialmente motivo de que esta relación estuviera excluida del ámbito de contrato de trabajo ${ }^{6}$, por considerarse que el vínculo que se creaba en la "casa" suponía una relación entre personas, al invadirse la intimidad familiar de la esfera de los sujetos del hogar y ello implicaba que se tratase de una prestación sometida a un régimen ajeno al propio contrato de trabajo. En la actualidad se sigue manteniendo esta característica ${ }^{7}$, siendo el ámbito íntimo, personal y confidencial en el que se desarrollan los servicios el que determina la opción legislativa de excluir este contrato del régimen general que afecta al contrato de trabajo ordinario y determina el alejamiento del concepto de empresario utilizado en la contratación laboral ordinaria que define el artículo 1.2 del Estatuto de los Trabajadores.

Es por ello, que se exige que el titular del hogar familiar y empleador sea una persona física, no entendiéndose que existe relación laboral especial cuando la prestación de servicios domésticos haya sido contratada por una persona jurídica, sea una empresa ordinaria, una empresa de trabajo temporal (art. 2.1.a) y b) del Real Decreto 1620/2011) o un organismo público ${ }^{8}$. No es por ello relación especial sino ordinaria la prestación de servicios de limpieza, cocina, etc. contratados por una empresa para desarrollarlos en una residencia de la que es titular ${ }^{9}$. Tampoco lo es cuando los contratantes son varios miembros de una misma familia que residen cada uno de ellos en un lugar distinto y contratan a una persona para atender al cuidado de otro familiar que está internado en

\footnotetext{
${ }^{5}$ STSJ País Vasco de 22 de septiembre de 2015, Rec. 1467/15.

${ }^{6}$ STS 27 de mayo de 1981, Rec. 2362/81.

${ }^{7}$ STS 21 de octubre de 2008, Rec. 4143/07.

${ }^{8}$ Sala Franco, T. La relación laboral especial del servicio del hogar familiar y el contrato de trabajo doméstico. Relaciones Laborales. T. I. núm. 4. 1986, pág. 289.

${ }^{9}$ STSJ Madrid de 25 de marzo de 2003, Rec. 5439/02.
} 
una residencia ${ }^{10}$ y tampoco cuando se prestan servicios para una comunidad formada por varios pisos unidos con elementos comunes (biblioteca, comedor social, etc.) ${ }^{11}$.

Nada debe objetarse a que la contratación del empleado al servicio del hogar familiar se realice con la intervención de un tercero (representantes del titular del hogar familiar o asesor de éste), siempre que su función se centre exclusivamente en la de asesoramiento y gestión administrativa de la documentación laboral y de Seguridad Social requerida para la contratación del personal al servicio del hogar familiar y posterior desarrollo del vínculo contractual, pues entre asesor y empleado doméstico no existe vínculo laboral alguno.

\section{LA CONTRATACIÓN MEDIANTE LA INTERMEDIACIÓN DE LOS SERVICIOS PÚBLICOS DE EMPLEO O DE LAS AGENCIAS DE COLOCACIÓN.}

La intervención en la contratación del empleado al servicio del hogar familiar de un tercero supone la inclusión en la fase inicial de ingreso al trabajo de un elemento que puede distorsionar en la práctica la eficacia jurídica de la relación laboral que une al titular del hogar familiar y al empleado que ha de prestar sus servicios por cuenta de aquél. La posibilidad de realizar la contratación a través de agentes intermediadores, unido a la opción de que ésta se produzca interviniendo una empresa de servicios domésticos ha abierto el debate sobre numerosas cuestiones en torno a las condiciones en las que el empleado/a de hogar ingresa en el mercado laboral que deben ser analizadas para clarificar cuándo la intervención de un tercero en la contratación de un empleado/a de hogar se realiza conforme a derecho y cuándo esta práctica debe considerarse fraudulenta o abusiva por contravenir la legislación laboral.

La contratación de los empleados al servicio del hogar familiar puede llevarse a cabo, además de por la contratación directa del empleador como se ha analizado en el epígrafe anterior, por la intervención de los servicios públicos de empleo y de las agencias de colocación debidamente autorizadas (art. 4.1 del Real Decreto 1620/2011). Esta intervención, que no se encontraba incluida en la anterior regulación (Real Decreto 1424/1985, de 1 de agosto, cuyo artículo 5 disponía que "no será de aplicación en el ámbito de esta relación laboral especial lo dispuesto en el artículo 16.1 del Estatuto de los Trabajadores, sin perjuicio dela prohibición de agencias privadas de colocación"), se encuadra dentro de las acciones de intermediación que ambos agentes tienen encomendadas por la legislación laboral (art. 32 del Real Decreto Legislativo 3/2015, de 23 de octubre, por el que se aprueba el Texto Refundido de la Ley de Empleo - LE en adelante- ), sin que ello suponga que puedan ser considerados como empleadores a los efectos del Real Decreto 1620/2011.

\footnotetext{
${ }^{10}$ STSJ País Vasco de 1 de septiembre de 2004, Rec. 1303/04.

${ }^{11}$ STSJ País Vasco 22 de septiembre de 2015, Rec.1467/2015. 
La intervención de las agencias de colocación en la contratación de los empleados de hogar debe ceñirse a lo regulado en el art. 4.1 del Real Decreto 1620/2011 en relación con lo dispuesto en el artículo 33 del Real Decreto legislativo 3/2015. Aparentemente, el régimen jurídico de las agencias de colocación es coherente con el artículo 15 del Convenio 189 OIT sobre el trabajo decente para las trabajadoras y los trabajadores domésticos, por cuanto prohíbe los eventuales abusos de estas agencias y prevé que los empleados de hogar puedan presentar las quejas o reclamaciones oportunas ${ }^{12}$. No obstante, en la práctica la legislación española en materia de agencias de colocación privadas se torna insuficiente para dar respuesta a la realidad actual, dado que el Real Decreto 1796/2010, de 30 de diciembre, por el que se regulan solo se regula el régimen jurídico de las que se dedican a labores de intermediación sin entrar a considerar otras opciones como la contratación de trabajadores a través de ellas. Debido a ello, la contratación de empleados de hogar por agencias de colocación es uno de los focos de incertidumbre jurídica por la ausencia de regulación legal que rija dicha contratación.

Con la legislación actual, el papel de las agencias de colocación solo puede ser uno a la hora de proceder a la contratación de los empleados al servicio del hogar familiar: el de intermediadores entre el titular del hogar familiar y el candidato a prestar dicho servicio. Y, para ello, deben cumplirse dos requisitos: En primer lugar, deben ser empresas debidamente autorizadas para actuar como tales (arts. 4.1 del Real Decreto 1620/2011 y art. 33.1 LE). Para ello, deben presentar una declaración responsable con carácter previo al inicio de su actividad, ante el Servicio Público de Empleo Estatal en el caso de que quiera actuar en más de una comunidad autónoma (art. 33.2 LE). Y, en segundo lugar, las agencias de colocación debidamente autorizadas deben tener como función principal la de intermediar en el mercado laboral, esto es, conectar las ofertas de trabajo con los trabajadores que buscan empleo (art. 33 LE) ${ }^{13}$.

Ambas notas caracterizadoras determinan que su intervención en el proceso de contratación de los empleados al servicio del hogar familiar debe ceñirse a la prestación de servicios de asesoramiento, consultoría y selección de las personas susceptibles de ser contratadas directamente como empleados/as de hogar por el cliente, que es el empleador o titular del hogar familiar. La labor de asesoramiento puede ser puntual, al inicio de la búsqueda y selección del candidato idóneo para el puesto requerido por el titular del hogar en el que se desempeñara finalmente el empleado sus servicios profesionales o puede extenderse en el tiempo, abarcando todo el tiempo que dure la relación laboral, en forma análoga a la que se realizan en las asesorías laborales con cualquier empresario, gestionado los trámites administrativos propios de esta contratación inicial y posterior desarrollo del vínculo contractual ${ }^{14}$. Esta labor se hace en ocasiones muy necesaria a tenor de la falta de conocimientos específicos en materia

\footnotetext{
${ }^{12}$ En este sentido se manifiesta Rodríguez Cardo, I.A. El nuevo sistema especial de empleados de hogar: una "revolución inconclusa". Civitas. Revista española de derecho del trabajo. № 158, 2013, pág. 61.

13 Véase por ejemplo las funciones que desempeña la agencia de colocación "SerHogarsystem" (https://www.serhogarsystem.com/servicio-domestico/) $\mathrm{o}$ "Casperfecta" (https://www.casaperfecta.es/empleadas-de-hogar.html)

${ }^{14}$ STSJ País Vasco 27 de octubre de 2015, Rec.1813/2015. ISSN: $2174-6419$

Lex Social, vol. 9, núm. 2 (2019)
} 
de contratación y relaciones laborales que suelen tener los titulares de los hogares familiares.

En relación con la actividad profesional que sin duda las agencias de colocación pueden desempeñar como empresas de selección de personal de servicio doméstico hay que puntualizar que puede abarcar una gran variedad de servicios; desde valorar los perfiles $\mathrm{y}$ aptitudes de los candidatos al puesto de trabajo hasta poner en relación los conocimientos y cualificación profesional de las personas trabajadoras con los requerimientos y características de los puestos de trabajo ofertados (art. 2.1 del Real Decreto 1796/2010, de 30 de diciembre, por el que se regulan las agencias de colocación). Si finalmente se realiza la contratación, la agencia de colocación no es responsable de la relación laboral pactada entre el cliente y el empleado/a de hogar en tanto que no es empleador, sino agente intermediador.

En el desarrollo de la labor de intermediación, tanto los servicios públicos de empleo como las agencias de colocación, están obligados a garantizar el principio de igualdad en el acceso al empleo, no pudiendo establecer discriminación alguna basada en motivos de origen, incluido el racial o étnico, sexo, edad, estado civil, religión o convicciones, opinión política, orientación sexual, afiliación sindical, condición social, lengua del Estado y discapacidad, siempre que los trabajadores se hallasen en condiciones de aptitud para desempeñar el trabajo o empleo de que se trate, tal y como dispone el art. 4.2 del Real Decreto 1620/2011. Además, en conexión con lo indicado en el art. 35 LE, la publicidad de las ofertas de empleo que se formulen para la contratación de personas para el servicio doméstico no podrá contener discriminación alguna basada en los motivos anteriormente mencionados.

De este modo, se infiere de la regulación legal, que las labores de intermediación sólo pueden realizarse por los agentes intermediadores identificados como tales por el ordenamiento laboral; esto es, servicios públicos de empleo, estatales o autonómicos y agencias de colocación debidamente autorizadas, que pueden ser personas físicas o jurídicas, según dispone el art. 33.2 LE. En relación con las empresas de trabajo temporal téngase en cuenta que pueden actuar como agencias de colocación si se ajustan a los requisitos exigidos por la LE; es decir, deben contar con autorización administrativa para realizar las funciones propias de las agencias de colocación y garantizar a los trabajadores la gratuidad en la prestación de servicios (art. 33.6 LE).

La contratación del empleado de hogar por terceros no autorizados para ello, como puede ser las agencias de colocación que no cuenten con la debida autorización, no comporta consecuencias en relación con la eficacia jurídica del contrato de trabajo. Dado que la contratación se realiza finalmente por el titular del hogar familiar, el contrato despliega todos sus efectos jurídicos con independencia de que la agencia de colocación o la empresa de trabajo temporal que haya intermediado en este sentido no cuente con la autorización precisa para ello; sin perjuicio de las responsabilidades administrativas previstas en el ordenamiento jurídico que puedan derivarse por dichas actuaciones. Tal y como dispone el artículo 16.1.a) del Real Decreto legislativo. 5/2000, 
de 4 de agosto, por el que se aprueba el Texto Refundido de la Ley sobre Infracciones y Sanciones en el Orden Social (LISOS, en adelante), será considerada falta muy grave: "Ejercer actividades de intermediación laboral, de cualquier clase u ámbito funcional, que tengan por objeto la colocación de trabajadores son haber presentado, con carácter previo a la actuación como agencia de colocación, una declaración responsable, de reunir los requisitos de la Ley 53/2003, de 16 de diciembre de Empleo (actualmente art. 33.1 LE), y su normativa de desarrollo". Igualmente lo será, en relación con la actuación de las empresas de trabajo temporal, "en el caso de las empresas de trabajo temporal que hubieran presentado declaración responsable para actuar como agencias de colocación de reunir los requisitos establecidos en la Ley 56/2003, de 16 de diciembre, de Empleo (actualmente LE), y su normativa de desarrollo, incumplir los mismos". En ambos casos, las sanciones que pueden imponerse por la autoridad laboral oscilan entre los 6.251 y los 187.515 euros.

\section{LA EXTERNALIZACIÓN DE ACTIVIDADES MEDIANTE EMPRESAS DE SERVICIOS DOMÉSTICOS Y LA CONTRATACIÓN IRREGULAR DE LOS EMPLEADOS AL SERVICIO DEL HOGAR FAMILIAR.}

\subsection{Estado de la cuestión.}

La inclusión en el Régimen General de la Seguridad Social de las personas que realicen tareas domésticas y que hayan sido contratados, no directamente por los titulares del hogar familiar, sino al servicio de empresas por la disposición adicional $17^{\mathrm{a}}$ de la Ley 27/2011, de 1 de agosto, sobre actualización, adecuación y modernización del sistema de Seguridad Social vino a poner sobre la mesa la problemática cuestión de si dicha regulación legal estaba dando carta de naturaleza a la contratación de empleados al servicio del hogar familiar por parte de empresas, contradiciendo así lo establecido en el art. 2 del Real Decreto 1620/2011. Esta posibilidad está siendo también utilizada por empresas autodenominadas "agencias de colocación" o "agencias de servicio doméstico" para proceder a la contratación irregular de empleados domésticos con aumento de la precarización en el empleo y en las condiciones laborales de estos trabajadores $^{15}$. En estos casos, las empresas de servicios o las pseudo-agencias de colocación se convierten en contratistas de servicios domésticos mediante la concertación con el titular del hogar familiar de un contrato de prestación de servicios por el que aquélla se compromete a facilitarle un empleado doméstico que prestará sus servicios en el seno del hogar familiar, pero bajo la dirección y retribución de la empresa de servicios. En estos casos, nos encontramos con la tesitura de determinar si estamos ante una verdadera contrata de servicios o ante una cesión ilegal de

\footnotetext{
${ }^{15}$ La asociación de trabajadoras del hogar ATH-LE de Bizcaia viene denunciando ante la Inspección de Trabajo sistemáticamente las actuaciones fraudulentas de agencias de colocación de trabajadoras de hogar (pueden consultarse algunas de las denuncias interpuestas por la asociación en la web de la misma: https://drive.google.com/file/d/1IspjE06mev_kGdaV5VRSMAkGZ1IWMWgy/view;

https://drive.google.com/file/d/0B9JmBJnkcwuIeDlCX2pQcjZOeUU/view;

https://docs.google.com/file/d/0B9JmBJnkcwuIOWF0ZE9iVFdWOVU/edit -consultado el 08/05/2019-). ISSN: $2174-6419$

Lex Social, vol. 9, núm. 2 (2019)
} 
trabajadores, prohibida en nuestro ordenamiento jurídico en virtud de los arts. 16.3 y 43 del Estatuto de los Trabajadores. Ambas opciones plantean numerosas cuestiones que deben analizarse para determinar la verdadera naturaleza de la relación existente entre el titular del hogar familiar, el empleado y la empresa de servicios y, en el supuesto de que se determine que se trata de una cesión encubierta de trabajadores, las consecuencias que el tráfico fraudulento de mano de obra comporta para cada uno de ellos.

Partimos en este análisis de las exclusiones contenidas en los apartados a) y b) del Real Decreto 1620/2011 que excluyen del ámbito de la relación laboral especial del servicio de hogar familiar las relaciones concertadas por personas jurídicas, sean de carácter civil o mercantil, y por empresas de trabajo temporal. Ello determina que la regulación contenida en la disposición adicional $17^{\text {a }}$ de la Ley 27/2011 puede parecer a primera vista innecesaria ${ }^{16}$, salvo que la intención del legislador fuera permitir la contratación de trabajadores domésticos por parte de empresas de servicios, eliminando así de forma tácita la existencia en este supuesto de una cesión ilegal de trabajadores ${ }^{17}$.

Otra cuestión que debe debatirse es en qué supuestos la persona contratada formalmente por la empresa de servicios, pero que presta servicios domésticos en el hogar familiar de un tercero, debe reputarse como trabajador de aquélla. Si la disposición adicional $17^{\mathrm{a}} \mathrm{de}$ la Ley 21/2011 va más allá de lo que se extrae de su literalidad, esto es, que quien no está contratado directamente por el titular del hogar familiar en el que presta servicios sino por una empresa debe encuadrarse en el Régimen General de la Seguridad Social, la disyuntiva que se plantea es si puede considerarse como ordinaria la relación existente entre la empresa de servicios domésticos y el empleado que presta servicios en el domicilio de un cliente que ha contratado a aquélla precisamente para que le facilite un trabajador que desempeñe labores domésticas en su hogar. Creemos que ello es del todo posible, tal y como refleja la disposición adicional $17^{\text {a }}$ de la Ley $21 / 2011$, cuya función no es dar cobertura legal a esta posibilidad sino indicar que esta opción es viable en la práctica, pero que en este caso no estaremos ante una relación laboral especial sino ordinaria, de ahí que el empleado se encuadre en el Régimen General de la Seguridad Social que es donde se encuadra su empleador. Una vez realizada la contratación de empleados de hogar a través de empresas de servicios, dicha contratación no es fraudulenta si se lleva a cabo mediante un contrato de trabajo ordinario y no mediante el contrato especial del servicio del hogar familiar.

Para tratar de dar respuesta a los interrogantes que la contratación irregular de empleados/as de hogar por parte de agencias de colocación genera en la práctica, debemos partir de lo ya expuesto con anterioridad en relación con la función que a éstas les compete en relación con el ingreso en el mercado laboral de los de empleados al servicio de hogar familiar: en primer lugar, pueden poner en contacto al empleador con posibles candidatos para cubrir las necesidades de labores domesticas que el hogar de

\footnotetext{
${ }^{16}$ De obviedad lo califica Beneyti Calatayud, D. La reforma de la jubilación y otras prestaciones. La Ley CISS. Wolters Kluvers. 2011.

${ }^{17}$ De esta opinión es López Gandía, J./ Toscani Gimenez, D. Las causas de la reforma de la Seguridad Social. Análisis prácticos de la Ley 27/2011. Ed. El Derecho. 2011, pág. 343. 
aquél requiera $\mathrm{y}$, en segundo lugar, puede auxiliar al empleador en las labores administrativas de gestión del contrato. Su actuación en este caso se limita a la mera intermediación y gestión laboral, no existiendo entre la agencia de colocación y el empleado de hogar ningún tipo de relación laboral y derivándose la ilegalidad e la intermediación solo en el caso concreto de que la agencia no cuente con la autorización administrativa correspondiente. Cualquier otra modalidad de contrato que vincule a la agencia de colocación con el titular del hogar familiar deberá pasar por el tamiz de la legalidad jurídica, sin poder determinarse a priori, si la relación existente entre ambos es un arrendamiento de servicios para la búsqueda y selección de personal o enmascara un supuesto de cesión ilegal de trabajadores. La cuestión se complica al ser una práctica habitual que las empresas de servicios domésticos se mimeticen como empresas de selección o agencias de colocación, en este último caso, sin la autorización exigida para intermediar y ello porque, bajo la apariencia de dichas empresas pretenden encubrir una práctica fraudulenta.

\subsection{Análisis de los supuestos problemáticos en torno a la contratación irregular por parte de las empresas de servicios.}

Son dos los supuestos problemáticos que la contratación de los empleados al servicio del hogar familiar plantea y que parten de la utilización fraudulenta por parte de las empresas de servicios domésticos de esta relación laboral especial, con la consiguiente desprotección y precarización de este colectivo de trabajadores, fuertemente feminizado.

El primero de ellos comienza con la firma efectiva del contrato laboral que da lugar a la relación laboral especial por parte del titular del hogar familiar para la realización de las labores domésticas definidas en el art. 1.4 del Real Decreto 1620/2011, pero en cuyo proceso de contratación ha intervenido una empresa de servicios domésticos (o una agencia de colocación o empresa de selección de trabajadores domésticos), que ha sido contratada por el titular del hogar familiar para que gestione, no solo la selección del empleado sino la gestión laboral del contrato de trabajo, esto es, el abono de los salarios, la ordenación del servicio prestado, la concreción de las vacaciones y los días de descanso, caracterizándose asimismo esta relación contractual entre empresa de servicios y cliente en que aquélla se compromete a suministrarle de forma permanente durante todo el tiempo del contrato entre ambas, empleados de hogar a demanda.

El segundo de los supuestos problemáticos es muy similar al primero: también existe un contrato de arrendamiento de servicios entre la empresa de servicios domésticos (o agencia de colocación o selección, etc.), para la selección y permanente suministro de trabajadores domésticos al cliente (el titular del hogar familiar), pero en este caso no se realiza el contrato laboral entre el titular del hogar familiar y el empleado, sino que éste es contratado por la propia empresa de servicios, si bien, la empresa de servicios domésticos es finalmente la que gestiona todo lo relacionado con la prestación laboral, abonando el salario, determinando los horarios y vacaciones y, de forma similar al primer supuesto, reemplazando al trabajador doméstico cuando el cliente así lo exija; 
normalmente previo despido del mismo por cualquier circunstancia relacionada con el servicio o con las capacidades o aptitudes del trabajador que no son las requeridas o exigidas por el cliente-titular del hogar familiar. Quizás este segundo supuesto problemático pueda parecer al inicio, carente de interés discursivo, toda vez que se trata de un arrendamiento de servicios entre una empresa y cliente que suministrará trabajadores, previamente contratados por la misma, para realizar unas funciones concretas en un lugar concreto determinado por el cliente.

Pero si analizamos los litigios planteados ante la jurisdicción social y ante la Inspección $^{18}$ de Trabajo por los trabajadores del sector de los empleados de hogar constataremos que, en muchos casos, la demandas se centran en que las empresas de servicios hace firmar a los trabajadores contratos laborales de carácter especial, sin indicarles o informales que lo que surge con su firma es una relación laboral ordinaria, no sujeta a las garantías y derechos reconocidos por el Real Decreto 1620/2011 a los empleados del servicio del hogar familiar, con la precarización que ello está suponiendo en la práctica.

Partiendo pues del carácter problemático, por lo abusivo de los hechos cometidos por las empresas de servicio en este sentido, nos detendremos a analizar con detalle cada uno de los supuestos planteados con el objetivo de visibilizarlos y determinar cuáles son las consecuencias que nuestro ordenamiento jurídico ha previsto para garantizar los derechos de los trabajadores sometidos a la contratación irregular.

El primero de los supuestos expuestos para su análisis parte de la firma efectiva del contrato laboral por parte del titular del hogar familiar, que puede ser indefinido o temporal, pero que incluye las "cláusulas específicas de trabajadores de servicio de hogar familiar" (como por ejemplo el tiempo de presencia, la pernoctación o no del trabajador, si se establecen o no prestaciones salariales en especie, etc.), pero en el proceso de ingreso al mercado laboral ha intervenido una empresa de servicios, con independencia de la denominación de ésta (ya lo hemos indicado anteriormente).

La determinación en estos casos de si nos encontramos ante una relación laboral especial amparada por el Real Decreto 1620/2011 o una relación ordinaria a la que resulta de aplicación exclusivamente el Estatuto de los Trabajadores, parte del análisis casuístico. Si los contratos de trabajo para la prestación del servicio de hogar se rubricaron entre el titular del hogar familiar y el trabajador/a, pero el desarrollo de la relación laboral indica la existencia de una relación ficticia entre ambos que parte de la mera plasmación de la firma del titular del hogar familiar en un contrato confeccionado y puesto a su disposición por la empresa de servicios, tendremos el primer elemento que debe inducirnos a considerar que nos entramos ante una contratación irregular del empleado/a de hogar. Las consecuencias jurídicas que este hecho conlleva para este último, en relación con sus derechos laborales, pasan por determinar si existe o no una relación laboral entre la empresa de servicios y el empleado/a de hogar.

${ }^{18}$ Ver nota 11. 
Como sabemos, en nuestro derecho positivo el contrato de trabajo resulta definido en el art. 1.1 del Estatuto de los Trabajadores, cuando al señalar el ámbito objetivo de dicha Ley, dispone que "será de aplicación a los trabajadores que voluntariamente presten sus servicios retribuidos por cuenta ajena y dentro del ámbito de organización y dirección de otra persona física o jurídica, denominada empleador o empresario".

Por otra parte, el art. 8.1 del mismo Estatuto de los Trabajadores consagra una presunción "iuris tantum" de existencia del mismo, al decir que "el contrato de trabajo... se presumirá existente entre todo el que presta un servicio por cuenta ajena y dentro del ámbito de organización y dirección de otro y el que lo recibe a cambio de una retribución a aquél". De la exégesis normativa se deduce que para que exista un contrato de trabajo ha de intercambiarse, entre el trabajador y el empresario, un trabajo dependiente y un salario garantizado ajeno a los riesgos de la empresa. Dependencia y ajenidad serán, así, los elementos esenciales de necesaria presencia en el contrato de trabajo $^{19}$. Por otra parte, el Tribunal Supremo ha reiterado ${ }^{20}$ que la determinación de si una relación "inter partes" tiene o no naturaleza laboral, no depende no de cómo la conciban ni de como la denominen las partes, ni de ninguna resolución o decisión administrativa, sino que tan solo compete a los órganos judiciales, que han de atender al verdadero contenido obligacional para determinar la verdadera naturaleza de aquella.

Trasladando esta jurisprudencia al caso que nos ocupa, debe analizarse cuál es la realidad de la prestación del servicio y para quién los prestan los trabajadores y en qué condiciones lo prestaban, para determinar con ello si se trata de una relación laboral ordinaria o especial.

Con independencia de que la empresa de servicios haya podido firmar con el cliente un contrato de arrendamiento de servicios por el que se compromete a realizar una selección del personal prestará servicios para los distintos clientes que puede consistir en el estudio y valoración del curriculum vitae y la realización de pruebas de personalidad y conocimiento y la comprobación de las referencias facilitadas al candidato y con independencia de que se haya formalizado entre el cliente-titular del hogar familiar un contrato al amparo del Real Decreto 1620/2011, el análisis casuístico determinará la verdadera naturaleza de la relación existente entre la empresa de servicios y el trabajador doméstico tras la valoración de dos elementos que, a nuestro juicio, son determinantes para determinarla y determinará quién deba responder por las actuaciones abusivas y las condiciones laborales precarias de las que pueda ser sujeto el empleado doméstico; dichos elementos son la dependencia y la ajenidad en la retribución.

La dependencia o subordinación del trabajo significa que el trabajador se encuentra en una situación de "sometimiento a la esfera organicista, rectora o disciplinaria del empresario", esto es, que quien organiza el trabajo, da las órdenes y sanciona en caso de

\footnotetext{
${ }^{19}$ SSTS de 9 de diciembre de 2004, Rec. 5319/2003; de 11 de marzo de 2005, Rec. 2109/2004; o de 18 de marzo de 2009, Rec. 1709/2007.

${ }^{20}$ Por todas, SSTS 14 de noviembre de 1983 y 10 de abril de 1984.
} 
incumplimiento de sus obligaciones es el empresario y no el trabajador ${ }^{21}$. Existen distintos grados de dependencia en el trabajo. Naturalmente, en cada contrato de trabajo, la dependencia posee una mayor o menor gradación, según las características de la actividad que se realice y el puesto de trabajo que se ocupe ${ }^{22}$. Así, existirá mayor dependencia en el contrato de embarco en la marina mercante o en el contrato de un peón de la construcción que en el contrato para trabajos a domicilio o en el contrato de un trabajador altamente cualificado o directivo o de un representante de comercio.

En el caso de los empleados/as de hogar, la constatación de que existe dependencia entre la empresa de servicios domésticos (agencia colocación, empresa de selección de personal doméstico, etc.) y el trabajador/a derivaría de las siguientes circunstancias: estipulación por parte de la empresa de las condiciones esenciales en las que deberá quedar sujeta la prestación del servicio doméstico por parte de la trabajadora; el desarrollo de las tareas domésticas en los domicilios de los clientes o en los centros que ellos les indiquen; la puesta en conocimiento por el cliente de forma obligatoria de cualquier modificación en la prestación del servicio o la intervención de la empresa en la justificación de las horas de servicio y, de forma determinante, la sustitución del trabajador/a por otro/a en el caso de que no se pudiera prestar el servicios por baja voluntaria, extinción de la relación de trabajo o en caso de baja médica o disfrute de vacaciones $^{23}$. No creemos que estos indicios puedan quedar desvirtuados porque la prestación de servicios pueda estar modulada u organizada por concretas instrucciones del titular del hogar, puesto que ello solo afectaría al modo de la prestación del servicio, ni porque existiera pernoctación en el domicilio del cliente.

El segundo elemento clave para determinar que existe una relación laboral entre la empresa de servicios y el empleado/a de hogar es la ajenidad en la retribución. La ajenidad significa que la retribución se encuentra garantizada con independencia de los beneficios o pérdidas del empresario, esto es, con independencia de los riesgos empresariales $^{24}$. El trabajador, por su prestación laboral dependiente, percibirá del empresario, en cualquier caso, una compensación económica garantizada, sin quedar afectada por el riesgo de pérdida o mayor onerosidad en la ejecución de aquella (deterioro o destrucción del trabajo realizado o defectos en la comercialización del producto, por ejemplo). El trabajador cumple así con la mera puesta a disposición del empresario y no porque se haya producido el resultado pretendido por éste, ya que se trata de una obligación de medio y no de resultado. En el caso del servicio de hogar familiar, el empleado/a de hogar presta servicios retribuidos, como cualquier trabajador. Pero la delimitación de quién le retribuya es clave para determinar quién es su empresario. Los indicios que determinan la existencia de ajenidad en la retribución son

\footnotetext{
${ }^{21}$ Por todas, STS de 2 de julio de 1996, Tol 236721.

${ }^{22}$ SSTS de 17 de noviembre de 2004, Rec. 6006/2003 o de 20 de noviembre de 2007, Tol 1214267.

${ }^{23}$ Indicios todos ellos que han sido tenidos en cuenta por la doctrina de los TSJ: SSTSJ de Barcelona de 13 de marzo de 2012, Rec. 5971/2011; Valladolid, de 29 de julio de 2013, Rec. 948/2013; Burgos, de 25 de marzo de 2014, Rec. 187/2014; Bilbao, de 22 de septiembre de 2015, Rec. 1467/2015

${ }^{24}$ SSTS de 22 de abril de 1996, Rec. 2613/1995 o de 31 de marzo de 1997, Rec. 3555/1996. 
los siguientes: abono del salario por parte de la empresa de servicios; realización de diligencias para su alta en Seguridad Social; abono de las cuotas de Seguridad Social.

Estos indicios no pueden quedar desvirtuados por la incorporación en los contratos de arrendamientos de servicios entre la empresa y el cliente cláusulas del siguiente tenor: "La empresa se compromete con el cliente a realizar la gestión del pago del salario a la empleada de hogar, en nombre del cliente firmante entre los días 5 y 10 de cada mes por lo que se exige el cumplimiento puntual de la reposición de fondos por parte del

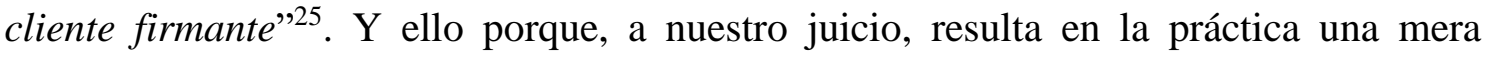
ficción ya que a pesar de que formalmente el pago lo hace el titular del hogar familiar, indirectamente dichos pagos quedarían incluidos en la factura que la empresa entrega mensualmente al cliente por los servicios prestados, entre los que se incluye la retribución de los trabajadores. Se trataría de una pantalla jurídica que, en realiza, enmascara una relación laboral ordinaria entre la empresa de servicios y el trabajador/a doméstico.

En consecuencia, la constatación de la existencia de estos indicios de dependencia y ajenidad en la relación laboral de los empleados/as de hogar condiciona su naturaleza jurídica, tornándose en ordinaria, regulada por el Estatuto de los Trabajadores y no por el Real Decreto 1620/2011, así como hace derivar ciertas responsabilidades para quien ostenta la condición de empresario real, esto, es, la empresa de servicio (agencia de colocación, de selección, etc.) e incluso para el titular del hogar familiar que firma el contrato fraudulento, por no actuar conforme a lo esperado para un empleador. En estos casos, creemos posible considerar que estamos ante un supuesto de cesión ilegal de trabajadores, amparado por el art. 43 del Estatuto de los Trabajadores, pudiendo exigirse responsabilidades tanto a la empresa cedente como al titular del hogar familiar. La firma del contrato de trabajo con las "cláusulas específicas de trabajadores de servicio de hogar familiar" por parte del cliente de la empresa de servicios determinan que, a efectos del ordenamiento jurídico laboral es el empleador del trabajador que prestará el servicio en el ámbito del hogar familiar (art. 1.3 del Real Decreto 1620/2011). Son dos pues, las empresas implicadas en el tráfico prohibido que caracteriza la aplicación de las garantías reguladas por el art. 43 del Estatuto de los Trabajadores: por una parte, la empresa de servicios y por otra, el titular del hogar familiar que se convierte en empleador mediante la firma del contrato. Como se recordará, este precepto indica que "la contratación de trabajadores para cederlos temporalmente a otra empresa solo podrá efectuarse a través de empresas de trabajo temporal debidamente autorizadas en los términos que legalmente se establezcan". Los elementos que, a nuestro juicio, determinan que podamos aplicar en este supuesto concreto, el artículo 43 del Estatuto de los Trabajadores: en primer lugar, que la cesión de trabajadores se realiza sin que medie una empresa de trabajo temporal, dado que la empresa de selección de servicios (agencia de colocación, etc.) no lo es (analizaremos a continuación las consecuencias

${ }^{25}$ Clausula incluida en el contrato de arrendamiento de servicios entre la empresa Servillar Gestión de Servicios de Personal, S.L. y los titulares de los domicilios donde prestaban servicios las empleadas de hogar, objeto de análisis en la STSJ de Barcelona, de 13 de marzo de 2012, Rec. 5971/2011. 
puede conllevar que lo sea); en segundo lugar, que si bien es el titular del hogar familiar el que firma el contrato de trabajo y no la empresa de servicios, los indicios de laboralidad indicados anteriormente (dependencia y ajenidad en la retribución) demuestran que ésta es el empresario efectivo y que es el que pone a disposición de aquél los trabajadores.

La actuación fraudulenta por parte de la empresa de servicios domésticos y del titular del hogar familiar firmante del contrato de trabajo determina en la práctica una suerte de consecuencias jurídicas para el empleado/a de hogar y de responsabilidades para los empresarios implicados en la contratación irregular. La consideración de la empresa de servicios como empresario-empleador real del empleado/a de hogar determina que, en aplicación del artículo 8 del Estatuto de los Trabajadores, adquiere la condición de trabajador de dicha empresa, si bien su relación laboral no es ya especial, con las desventajas que ello supone. No obstante, debido a que también han sido objeto de una cesión ilegal de trabajadores, dicho derecho les viene reconocido por el art. 43.4 del Estatuto de los Trabajadores, que les permite adquirir la condición de fijos, a su elección, en la empresa cedente o en la cesionaria; esto es, ser formar parte de la plantilla de la empresa de servicios, con las consecuencias que ello comporta en el momento en el que finalice el contrato entre ésta y el cliente-titular del hogar familiar a efectos de extinción del contrato inicialmente firmado. La jurisprudencia admite que en los casos en los que se aplica el art. 8.1 del Estatuto de los Trabajadores, como es el que analizamos, la opción por la empresa cesionaria será automática ${ }^{26}$, por lo que no cabe la opción de que el titular del hogar familiar sea admitido como empleador una vez constatada la existencia de indicios de laboralidad respecto de la empresa de servicios. El derecho de opción se ejerce mientras dure la cesión y no con posterioridad ${ }^{27}$, sin que sea necesario que transcurra ningún plazo de tiempo para que el trabajador cedido tenga derecho de opción; esto es, tiene ese derecho con independencia de la duración de la cesión.

Los derechos y obligaciones de los trabajadores cedidos serán los que correspondan en condiciones ordinarias a un trabajador que preste servicio en el mismo o equivalente puesto de trabajo. Computándose la antigüedad desde el inicio de la cesión ilegal (art. 43.4 ET) [S.T.S. de 3 de febrero de 2000 (Tol 57422)].

Los derechos y obligaciones del trabajador que opte por convertirse en fijo de la empresa cesionaria serán los aplicables a los trabajadores de dicha empresa, incluso aunque estos derechos y obligaciones fueran inferiores a los aplicables a la empresa cedente (S.T.S., en unificación de doctrina, de 31 de octubre de 1996, Ar/8166).

También tendrá derecho a las diferencias salariales si la retribución de la empresa cesionaria fuese mayor que la cedente (S.T.S., en unificación de doctrina, de 4 de julio de 2013, Rec. 2192/2012).

\footnotetext{
${ }^{26}$ STS de 17 de enero de 1991, Ar/58.

${ }^{27}$ STS de 11 de septiembre de 1986, Ar/4953. 
En clave sancionadora, el ordenamiento laboral prevé tres clases de responsabilidad en la que incurren los empresarios implicados en la cesión ilegal de trabajadores y que, desde luego, resulta predicable en su aplicación a las empresas de empresa de servicios, agencia de colocación, etc. y al titular del hogar familiar implicados en el tráfico prohibido; responsabilidades que son de orden contractual, administrativo y penal.

En primer lugar, la asunción de responsabilidad contractual por parte de los empresarios deriva de lo dispuesto en el art. 43.3 del Estatuto de los Trabajadores, los cuales habrán de responder de forma solidaria de las obligaciones contraídas con los trabajadores y con la Seguridad Social (art. 43.3 ET), siempre que subsista la situación de cesión ilegal $^{28}$. Dicha responsabilidad se extiende a todas las obligaciones laborales y no solo a las salariales, así como a las de Seguridad social (cotizaciones y prestaciones, en su caso, por falta de afiliación, alta y cotización).

En segundo lugar, la empresa de servicios domésticos (cualquiera que sea su denominación -agencia de colocación, empresa de selección del personal doméstico, etc.-) y el titular del hogar familiar incurren en responsabilidades de orden administrativo, por cuanto sus actuaciones están tipificadas como infracción por la LISOS. La cesión ilegal de empleados/as de hogar se encuentra tipificada como una infracción muy grave por el artículo 8.2 LISOS, lo cual genera una responsabilidad administrativa que debe imponerse a ambos empresarios ${ }^{29}$. Junto a ello, también pueden exigirse responsabilidad a la empresa de servicios domésticos por la realización de la conducta tipificada como infracción grave en el art. 7.2 de la LISOS: "la transgresión de la normativa sobre modalidades contractuales, contratos de duración determinada y temporales, mediante su utilización en fraude de ley o respecto a personas, finalidades, supuestos y límites temporales distintos de los previstos legal, reglamentariamente, o mediante convenio colectivo cuando dichos extremos puedan ser determinados por la negociación colectiva". En esta tipificación estaría comprendida la actuación de la empresa de servicios de entregar al cliente el contrato de trabajo de servicio de hogar familiar por incurrir en un supuesto de fraude de ley. Según dispone el art. 6.4 del Código Civil, el fraude de ley se produce cuando se realiza un acto no negocio jurídico amparándose en una norma, denominada ley de cobertura, con la finalidad de alcanzar ciertos objetivos que, no siendo los propios de esa norma, sean además contrarios a otra ley o al ordenamiento jurídico.

En el caso del contrato laboral que da origen a la relación especial del servicio de hogar familiar el fraude vendría de la mano de utilizar para otras finalidades distintas a las legalmente establecidas, cuales son, que se utilice para que el titular del hogar familiar contrate a un empleado que, dependientemente y por su cuenta preste servicios retribuidos en el ámbito del hogar familiar (art. 2.2 del Real Decreto 1620/2011); finalidad que queda desvirtuada cuando se utiliza para otra finalidad, haciendo firmarlo al cliente a sabiendas que éste no es el empresario real del trabajador doméstico. Debe tenerse en cuanta que el ilícito no se centra en la infracción de determinadas

${ }^{28}$ STS de 14 de septiembre de 2009, Tol 1627771.

${ }^{29}$ STS, u.d., de 31 de octubre de 1990, Ar/8166. 
formalidades a la hora de concertar el contrato; no es la ausencia de forma lo que se sanciona administrativamente, sino la intención empresarial de eludir el mandato de la norma legal. Así lo ha mantenido el Tribunal Supremo para el que el fraude de ley "es algo más que la simple omisión de determinadas formalidades en la configuración de la relación jurídica, envolviendo en todo caso, una decidida y patente voluntad de eludir el mandato imperativo de la norma legal, obviando la realización de su propio objetivo o finalidad"30.

En definitiva, para que la transgresión de la normativa sea considerada como infracción administrativa debe estar presidida por la voluntad de burlar la norma; de ahí que no resulte suficiente admitir su existencia, la de la infracción, por meros defectos en la contratación, por ejemplo, si ello no llegar a ser demostrativo de una verdadera voluntad de parte, tendente a burlar el imperativo legal. Pero creemos que el mero hecho de redactar el contrato y entregárselo al cliente para que lo firme y se convierta en el empleador ficticio del empleado/a de hogar es indicio más que suficiente de la intencionalidad de burlar la normativa legal que regula esta modalidad contractual.

Por último, la cesión ilegal de empleados/as de hogar también hace derivar responsabilidades penales. Ambos empresarios (empresa de servicios y titular del hogar familiar) pueden incurrir en el delito de tráfico ilegal de mano de obra, sancionado con las penas de prisión de seis meses a tres años y multa de seis meses a doce meses, por realización de las actuaciones delictivas previstas en los artículos 311.1 y 312.1 Código Penal ("los que, mediante engaño o abuso de situación de necesidad impongan a los trabajadores a su servicio condiciones laborales o de Seguridad Social que perjudiquen, supriman o restrinjan los derechos que tengan reconocidos por disposiciones legales, convenios colectivos o contrato individual" y "los que trafiquen de manera ilegal con mano de obra").

Para puntualizar el supuesto referenciado anteriormente sobre qué ocurre en el supuesto de que la empresa de servicios sea una empresa de trabajo temporal, ha de advertirse que ello es perfectamente posible en nuestro ordenamiento jurídico. De hecho, si los trabajadores que han de desempeñar servicios domésticos son contratados por una empresa de trabajo temporal para cederlos a otras empresas o clientes con los que media un contrato de arrendamiento de servicios, nada hay en ello objetable ni digno de mención. No existirá ningún inconveniente en que los clientes fueran titulares de hogar que bien pudieran haber contratado directamente a un empleado/a de hogar por la vía del Real Decreto 1620/2011, pero no siendo esta su opción, pueden contratar a una empresa de trabajo temporal para que le realice tal servicio. En este caso, los trabajadores domésticos contratados por la empresa de trabajo temporal, cuya relación laboral es ordinaria por cuanto no se han seguido los trámites establecidos en el Real Decreto 1620/2011 en la medida en que el contrato no se ha realizado un "empleador" sino por una empresa (ver exclusiones en art. 2), tienen los derechos regulados por la Ley 14/1994 que regula las empresas de trabajo temporal. No se trata en este caso de un

${ }^{30}$ STS de 4 de abril de 1990, RJ 3104/1990. 
supuesto de cesión ilegal de trabajadores, sino de una cesión legal de trabajadores domésticos que prestarán servicios para los clientes que previamente han contratado a la empresa de trabajo temporal para que les envíen trabajadores a sus hogares, única opción permitida por el artículo 43.1 del Estatuto de los Trabajadores.

El segundo de los supuestos problemáticos que analizaremos en este apartado se centra en determinar si es conforme al ordenamiento jurídico la realización de un contrato de arrendamiento de servicios entre la empresa de servicios domésticos (o agencia de colocación o selección, etc.) y el trabajador que desempeñará las labores domésticas en el hogar familiar del cliente. No existe en este caso contrato laboral entre trabajador y titular del hogar familiar, sino que éste es mero cliente de la empresa de servicios y beneficiario de los servicios del trabajador enviado a realizar las tareas propias del hogar, al cuidado de miembros de la familiar o a cualquier otra tarea que forme parte del conjunto de tareas domésticas como guardería, jardinería, conducción de vehículo y otros análogos. Las dudas surgen ahora en torno a la existencia en estos casos de una subcontratación de obra o servicio o una relación laboral especial de empleados de hogar ${ }^{31}$.

La opción del titular del hogar familiar de no contratar directamente los servicios de un empleado de hogar, sino los de una empresa que le ofrezca los mismos servicios que pudiera desempeñar aquél no plantea inconveniente, formalizándose en tal caso un contrato de arrendamiento de servicios domésticos entre la empresa de servicios y el titular del hogar familiar, que se convierte en cliente de la misma. La empresa de servicios es la encargada de gestionar todo lo relacionado con la prestación laboral, puesto que es el empresario de trabajador doméstico, abonando el salario, determinando los horarios y vacaciones $\mathrm{y}$, de forma similar al primer supuesto, reemplazando al trabajador doméstico cuando el cliente así lo exija; normalmente previo despido del mismo por cualquier circunstancia relacionada con el servicio o con las capacidades o aptitudes del trabajador que no son las requeridas o exigidas por el cliente-titular del hogar familiar. Lo que no sería posible sería calificar la relación laboral entre la empresa y la persona que realizará las labores domésticas como relación especial de servicio del hogar familiar, dado que el contrato no se firma con el titular del hogar familiar, como exige el art. 1.2 del Real Decreto 1620/2011, existiendo por lo tanto una relación labora ordinaria, amparada por lo establecido en el Estatuto de los Trabajadores.

Por lo que respecta a los derechos de los trabajadores contratados por la empresa de servicios domésticos, no les resulta de aplicación lo dispuesto en el Real Decreto 1620/2011, ni en los convenios colectivos que puedan, en su caso, negociarse a su amparo, al no resultar amparados por los mismos. Tampoco resultan amparados por los derechos reconocidos a los trabajadores en caso de subcontratación de servicios por el artículo 42 del Estatuto de los Trabajadores, dado que no se reúnen los requisitos que este precepto exige para la extensión de responsabilidad a las empresas que participan

\footnotetext{
${ }^{31}$ Circunstancia difícilmente imaginable para algunas resoluciones judiciales, como, por ejemplo, la STSJ de Barcelona, de 14 de octubre de 2008, Rec. 4666/2008.
} 
en la subcontratación. El primero de ellos sería la ausencia de dos empresarios, uno de los cuales contrata a otro la realización del servicio objeto del contrato. En el caso de la contratación de las empresas de servicios domésticos, el cliente, el titular del hogar familiar, no es una empresa sino un usuario del servicio. Podría argumentarse que el Real Decreto 1620/2011 equipara al titular del hogar familiar al empresario, pero ello choca frontalmente con el hecho cierto de que, en estos casos, no puede ser considerado empleador por cuanto no ha sido el encargado de contratar a la persona que prestará en su hogar el servicio contratado. El cliente no es empresario, no es empleador y no existen dos empresas, por lo que no concurre la primera nota exigida por el artículo 42 del Estatuto de los Trabajadores para su aplicación: "empresarios que contraten o subcontraten con otros".

El segundo requisito exigido por el citado precepto y que permitiría conferir a los trabajadores contratados por las empresas de servicios domésticos de los derechos reconocidos por el artículo 42 Estatuto de los Trabajadores es la existencia de la "propia actividad"; esto es, "las actividades del ciclo productivo, a diferencia de las actividades indispensables no inherentes a dicho ciclo, se incorporan al producto o resultado final de la empresa o entidad comitente, tanto si son realizadas directamente como si son encargadas a una empresa contratista, justificando así la responsabilidad patrimonial de la empresa o entidad comitente respecto de los salarios de los trabajadores empleados en la contrata"32. La propia especialidad de las funciones a desarrollar por los trabajadores domésticos rechaza la aplicabilidad de este elemento, dado que se ha admitido por la jurisprudencia como propia actividad la "actividad indispensable", de suerte que integrarán el concepto, además de las que constituyen el ciclo de producción de la empresa, todas aquellas que resulten necesarias para la organización del trabajo. Es decir, las tareas que se corresponden con el objeto de la "actividad principal" desarrollada por la empresa principal o contratante (las tareas que se corresponden con el "ciclo productivo" o que "se incorporan al producto o resultado final") o las tareas que tengan carácter "absolutamente esencial" para su ejecución ${ }^{33}$; pautas todas ellas muy alejadas de tareas domésticas.

\section{CONCLUSIONES Y PROPUESTAS PARA MEJORAR LA CONTRATACIÓN IRREGULAR DE LOS TRABAJADORES AL SERVICIO DEL HOGAR FAMILIAR.}

La contratación irregular de los trabajadores al servicio del hogar familiar por parte de empresas de servicios, enmascaradas bajo la apariencia de empresas de selección de personal doméstico o "pseudo-agencias de colocación" comporta en la práctica una suerte de desgracia para este colectivo debido a la ineficacia de la legislación vigente para aportar soluciones eficaces que pongan freno a dichas prácticas fraudulenta. La

${ }^{32}$ STS, u.d., de 21 de febrero de 2018, Rcud. 251/2016, reiterados en la STS de 9 de mayo de 2018, Rec. $3535 / 2016$.

${ }_{33}$ STS, u.d., de 18 de enero de 1995 (Tol 237082), de 29 de octubre y de 24 de noviembre de 1998 , Ar/9049 (Tol 47328) o de 22 de noviembre de 2002 (Tol 230291).

ISSN: $2174-6419$

Lex Social, vol. 9, núm. 2 (2019) 
utilización de aplicaciones y plataformas de trabajo colaborativo, en las que el cliente puede solicitar a la carta los servicios de un empleado/a de hogar están frivolizando la actividad laboral de estos trabajadores, siendo víctimas de la precariedad laboral y del recorte de derechos laborales.

La protección que el ordenamiento jurídico español brinda a los trabajadores del sector doméstico es todavía muy limitada, en la medida en que no existe una normativa unificadora por parte de la Unión Europea y el Convenio nº 189 de 2011 sobre el trabajo decente para las trabajadoras y los trabajadores domésticos de la OIT no ha sido ratificado aún por España ${ }^{34}$. A pesar de que dicho Convenio no incluye ningún aspecto relacionado con el con el ingreso en el mercado laboral de estos trabajadores, más allá de la eliminación de la discriminación en materia de empleo y ocupación, sí obliga a los Estados firmantes a adoptar las medidas necesarias para asegurar que los trabajadores domésticos gocen de una protección efectiva contra toda forma de abuso (art. 5 Convenio OIT $\mathrm{n}^{\circ}$ 189. 2011).

No cabe duda de que la contratación irregular es una forma de abuso; no es una fórmula que determine la informalidad de los servicios prestados por los empleados/as de $\operatorname{hogar}^{35}$, sino una práctica abusiva y fraudulenta que ralla en muchos casos la cesión ilegal de trabajadores. Como hemos analizado, los supuestos en los que una empresa de servicios, con independencia de cuál sea su denominación ("agencia de colocación", "agencia de colocación de servicios domésticos", "empresa de selección de personal doméstico", ...), tiene como actividad la gestión de empleados de hogar, gestión que se realiza mediante la suscripción de contratos de arrendamiento de servicios con el titular del hogar familiar y usuario del servicio, son indiciarios de la inexistencia de relación laboral especial con la consiguiente limitación de derechos de los trabajadores. Si a ello se une que la función de la empresa no se centra solo en seleccionar el personal encargado de la asistencia doméstica, sino que también se obliga a mantener dicha asistencia, gestionando el alta y el pago de cuotas a la Seguridad Social, así como el pago directo al empleado de hogar seleccionado finalmente ${ }^{36}$, estaremos lejos de considerar a esta relación laboral como especial, existiendo, como no podía ser de otra manera, una relación laboral ordinaria entre la empresa de servicios y el empleado al servicio del hogar familiar, lo que impediría aplicar a la misma lo dispuesto en el Real Decreto $1620 / 2011^{37}$, con los perjuicios que ello comporta para el propio trabajador que ve mermados sus derechos.

\footnotetext{
${ }^{34}$ Para un análisis en profundidad del Convenio 189 vid. Vela Díaz, R. La promoción internacional de un trabajo decente para las personas empleadas de hogar: el Convenio 189 de la OIT sobre los trabajadores domésticos. Revista del Ministerio de Empleo y Seguridad Social. № 127.

35 OIT. Formalización del trabajo doméstico. Nota de información 10. Disponible en: https://www.ilo.org/wcmsp5/groups/public/---ed_protect/---protrav/--travail/documents/publication/wcms_574115.pdf (consultado el 09/05/19).

${ }^{36}$ Así, se ha admitido por el TSJ de Burgos, en sentencia de 25 de marzo de 2014, Rec. 187/2014, que concurría los requisitos de ajenidad y dependencia cuando era la empresa de servicios la que pagaba a las empleadas de hogar, por ella contratadas.

${ }^{37}$ Esta es la situación analizada por al STSJ Valladolid, de 29 de septiembre de 2013, rec. 948/2013, en la que la empresa AFOSE Zamorana de Servicio Social, S.L. contrata a una empleada de hogar para prestar servicios para un cliente, al amparo de lo dispuesto en la disposición adicional $17^{\mathrm{a}}$ de la Ley $27 / 2011$, de 1 ISSN: $2174-6419$ Lex Social, vol. 9, núm. 2 (2019)
} 
Lamentablemente, los mecanismos legales que podían restituir en mayor o menor medida esta situación se muestran ineficaces, toda vez que está concebidos para situaciones distintas, como es el caso de las garantías ofrecidas por los artículos 42 y 43 del Estatuto de los Trabajadores, no aplicables directamente a todos los supuestos de contratación irregular de empleados/as de hogar. Tampoco la LISOS muestra un diseño legal que garantice la intervención de la Inspección de Trabajo ante prácticas abusivas en materia de contratación de empleados/as de hogar. Las actuaciones tipificadas como infracciones tampoco están diseñadas desde la óptica de las necesidades de este sector de trabajadores por lo que difícilmente son eficaces para erradicar una práctica que se nos antoja cada vez más extendida.

En definitiva y para concluir con nuestro análisis creemos necesaria una reforma legislativa que atienda a las especiales dificultades a las que se enfrentan los empleados/as de hogar a la hora de acceder al mercado laboral. Debe atenderse a las nuevas formas de contratación, que puedan provenir de empresas de servicios o de plataformas digitales o de economía colaborativa, las cuales dificultan al inicio de la relación laboral la determinación de quién deba ser considerado como empleador. Ello lleva a una precarización de las condiciones laborales de los trabajadores de este sector productivo precisamente porque las fórmulas existentes para la contratación, que son las reguladas en el Real Decreto 1620/2011 no cumplen con las exigencias reales que se demandan en la actualidad en materia de empleo doméstico. La regulación legal que dota de carácter especial al trabajo desempeñado por los empleados/as de hogar y que les brinda una suerte de derechos y garantías específicos en atención a dicha especialidad debe amparar otros supuestos en los que no sólo el titular del hogar familiar puede ser empleador; debe posibilitarse que también puedan ser consideradas empleadoras las empresas de servicios legalmente constituidas y sin ánimo fraudulento, con la consiguiente aplicación de los derechos del Real Decreto 1620/2011 a los trabajadores domésticos contratados por ellas para prestar servicios domésticos, fruto de la relación laboral especial que les une con la empresa de servicios.

Junto a la ampliación de los sujetos que puede ser empleadores en la relación laboral especial del servicio de hogar familiar, la opción por permitir que las agencias de colocación puedan ser empleadores de los trabajadores domésticos podría ser una opción viable que permitiría restringir los supuestos fraudulentos. En la línea de las garantías reconocidas a estos trabajadores por el art. 15.1 del Convenio 189 OIT, la reforma del Real Decreto 1796/2010, de 30 de diciembre, por el que se regulan las agencias de colocación podría incluir cláusulas que protegiesen efectivamente a los trabajadores domésticos contra las prácticas abusivas realizadas contra ellos por las agencias de empleo privadas y, en aplicación de lo dispuesto en el citada Convenio 189 de la OIT, las siguientes: a) asegurar la existencia de un mecanismo y procedimientos adecuados para la investigación de las quejas, presuntos abusos y prácticas fraudulentas

de agosto. El Tribunal califica la relación entre la empresa y la trabajadora de hogar recurrente como relación laboral oReal Decretoinaria, sin entrar lamentablemente a valorar si la citada empresa podía o no proceder a realizar dicha contratación. 
por lo que se refiere a las actividades de las agencias de empleo privadas en relación a los trabajadores domésticos; b) concretar las medidas necesarias y apropiadas para proporcionar una protección adecuada y prevenir los abusos contra los trabajadores domésticos contratados. Se incluirán las leyes o reglamentos en que se especifiquen las obligaciones respectivas de la agencia de empleo privada y del hogar para con el trabajador doméstico y se preverán sanciones, incluida la prohibición de aquellas agencias de empleo privadas que incurran en prácticas fraudulentas y abusos; c) considerar, cuando se contrate a los trabajadores domésticos en un país para prestar servicio en otro país, la concertación de acuerdos bilaterales, regionales o multilaterales con el fin de prevenir abusos y prácticas fraudulentas en la contratación, la colocación y el empleo.

En previsión de abusos y mientras que esta nueva legislación sobre agencias de empleo privadas no se lleve a término o de forma complementaria a la misma, debe abordarse por el legislador la tarea de actualizar los parámetros que dan lugar a entender que existe tráfico prohibido de mano de obra (mediante reforma del artículo 43 del Estatuto de los Trabajadores) para incluir aquellos supuestos en los que interviene de forma fraudulenta una empresa de servicios cuyo único objetivo en la cesión de trabajadores doméstico a sus clientes. Y, por último, dado que la denuncia ante la Inspección de Trabajo es en muchos casos la única opción que tiene el empleado/a de hogar para reivindicar sus derechos, deben clarificarse las infracciones relacionadas con este sector de actividad, incluyéndose en la normativa administrativa sancionadora la tipificación de conductas que sólo se producen en este sector de actividad profesional, como es el caso de responsabilidad en caso de cesión ilegal de trabajadores por "pseudo-agencias de colocación" o de empresas de selección que enmascaran que enmascaran la contratación de empleados de hogar bajo la apariencia de una contrata de obra y servicio.

A continuación, proponemos algunas líneas directrices que deberán informar una revisión de la legislación laboral, para amparar a los trabajadores del servicio del hogar familiar.

En primer lugar, abogamos por una ampliación de los sujetos que pueden ser considerados como empleadores a los efectos de la aplicación de los derechos reconocidos en el Real Decreto 1620/2011, extendiendo esta opción a las empresas de servicios domésticos e incluso a las empresas de trabajo temporal. Debe por ello eliminarse la restricción contenida en el art. 2.1,a) y b) de dicha norma legal.

En segundo lugar, ratificación del Convenio 189 OIT.

En tercer lugar, regulación de la contratación de trabajadores domésticos por parte de agencias de empleo privadas, con las garantías diseñadas por el Convenio 189 OIT.

En cuarto lugar, debe procederse a la revisión de los parámetros que identifican la cesión ilegal de trabajadores. Debería incluirse un apartado 3.bis en el artículo 43 del 
Estatuto de los Trabajadores que incluyera como causas de cesión ilegal de trabajadores las siguientes:

1.- Cuando mediando un contrato de arrendamiento de servicio entre una empresa y el titular de hogar familiar, éste no ejerza las funciones inherentes a su condición de empresario o no retribuya él mismo al empleado de hogar.

2.- Cuando mediando la firma de un contrato de los regulados en el Real Decreto 1620/2011, la contratación del empleado de hogar sea de tal naturaleza que no permita la constatación de los elementos de laboralidad del artículo 1.1 del Estatuto de los Trabajadores o cuando la contratación del empleado de hogar encubra un supuesto de fraude de ley.

Por último. en el plano de las infracciones laborales, debería incluirse en la LISOS o de forma especial en el propio Real Decreto 1620/2011, dentro de un apartado dedicado a las infracciones y sanciones en materia de infracciones administrativas, la tipificación como graves o muy graves de las siguientes conductas:

1.- La actuación de las agencias de colocación que exceda de su labor intermediadora, contratando a trabajadores domésticos para ponerlos a disposición de un cliente, en cuyo hogar familiar prestará los servicios, sin que medie contrato laboral entre el titular del hogar familiar y el empleado/a de hogar.

2.- La contratación de empleados/as de hogar por parte del titular del hogar familiar para que preste servicio en el domicilio de otra persona o empresa.

Mejorar los mecanismos de inserción en el mercado laboral y eliminar toda forma de contratación informal o irregular de los empleados/as de hogar es un elemento imprescindible para garantizar un trabajo digno.

\section{BIBLIOGRAFÍA}

- Alvarez Montero, A. ¿Una misma "norma de empleo" para investigadores de élite, parados de larga duración y empleados de hogar?: precariedad (laboral) e improvisación (legal). Estudios financieros. Revista de trabajo y seguridad social: Comentarios, casos prácticos: recursos humanos. № $.416,2017$.

- $\quad$ Ayala del Pino, C. El contrato de trabajo de los empleados del hogar: ingreso, conservación y extinción. Revista General de Derecho del Trabajo y de la Seguridad Social, ISSN-e 1696-9626, Nº. 47, 2017

- $\quad$ Beneyti Calatayud, D. La reforma de la jubilación y otras prestaciones. La Ley CISS. Wolters Kluvers. 2011.

- Espuny i Tomás (coord.), García González (coord.), Bonet Esteva (coord.). Relaciones laborales y empleados del hogar: reflexiones jurídicas. Dykinson, 2014.

- $\quad$ Espuny i Tomás, M.J. "El servicio doméstico: la historia jurídica de una exclusión continuada”. Relaciones laborales y empleados del hogar: reflexiones 
jurídicas / coord. por María Jesús Espuny i Tomás, Guillermo García González, Margarita Bonet Esteva, 2014.

- García González, G. Derechos sociales y empleados del hogar: reformas jurídicas inaplazables para la dignificación del trabajo doméstico en España. Revista de derecho social. $\mathrm{N}^{\mathrm{o}} 77,2017$, págs. 83-104.

- López Gandía, J./ Toscani Giménez, D. Las causas de la reforma de la Seguridad Social. Análisis prácticos de la Ley 27/2011. Ed. El Derecho. 2011.

- Lousada Arochena, J. F. Normativa internacional contra la explotación humana y laboral en el trabajo doméstico: la ONU y la OIT. Lan Harremanak. N 39. 2018,

- Miñambres Puig, C. El nuevo régimen jurídico de los empleados del hogar familiar. Civitas. Revista española de derecho del trabajo. No 157, 2013, págs. 111-133.

- $\quad$ OIT. Formalización del trabajo doméstico. Nota de información 10.

- $\quad$ OIT. Seguimiento de la resolución sobre medidas encaminadas a hacer realidad el trabajo decente para las trabajadoras y los trabajadores domésticos en todo el mundo: informe sobre los progresos realizados. 2013.

- $\quad$ OIT. Informe "Impacto de las reformas legislativas en el sector del empleo del hogar en España”. 2016.

- $\quad$ Organización de las Naciones Unidas. Informe de la Relatora Especial sobre las formas contemporáneas de la esclavitud, incluidas sus causas y consecuencias, Sra. Gulnara Shahinian. 2010.

- $\quad$ Rodríguez Cardo, I.A. El nuevo sistema especial de empleados de hogar: una "revolución" inconclusa. Civitas. Revista española de derecho del trabajo. N 158, 2013, págs. 143-198.

- $\quad$ Sala Franco, T. La relación laboral especial del servicio del hogar familiar y el contrato de trabajo doméstico. Relaciones Laborales. T. I. núm. 4. 1986.

- $\quad$ Salcedo Beltrán, M.C. "La relación laboral especial del hogar familiar: ámbito de aplicación, contratación y extinción”. Relaciones laborales y empleados del hogar: reflexiones jurídicas / coord. por María Jesús Espuny i Tomás, Guillermo García González, Margarita Bonet Esteva, 2014.

- Sanz Sáez, C. La discriminación en contra de las empleadas de hogar como forma de manifestación de las discriminaciones múltiples. Revista de derecho social. $\mathrm{N}^{\mathrm{o}}$ 83, 2018, págs. 89-108.

- Vela Díaz, R. La promoción internacional de un trabajo decente para las personas empleadas de hogar: el Convenio 189 de la OIT sobre los trabajadores domésticos. Revista del Ministerio de Empleo y Seguridad Social. № 127. 